\title{
Pendulum Motion in Main Parachute Clusters
}

\author{
Eric S. Ray ${ }^{1}$ \\ MRI Technologies, Houston, TX, 77058 \\ Ricardo A. Machin ${ }^{2}$ \\ National Aeronautics and Space Administration \\ Johnson Space Center, Houston, TX
}

The coupled dynamics of a cluster of parachutes to a payload are notoriously difficult to predict. Often the payload is designed to be insensitive to the range of attitude and rates that might occur, but spacecraft generally do not have the mass and volume budgeted for this robust of a design. The National Aeronautics and Space Administration (NASA) Orion Capsule Parachute Assembly System (CPAS) implements a cluster of three mains for landing. During testing of the Engineering Development Unit (EDU) design, it was discovered that with a cluster of two mains (a fault tolerance required for human rating) the capsule coupled to the parachute cluster could get into a limit cycle pendulum motion which would exceed the spacecraft landing capability. This pendulum phenomenon could not be predicted with the existing models and simulations. A three phased effort has been undertaken to understand the consequence of the pendulum motion observed, and explore potential design changes that would mitigate this phenomenon. This paper will review the early analysis that was performed of the pendulum motion observed during EDU testing, summarize the analysis ongoing to understand the root cause of the pendulum phenomenon, and discuss the modeling and testing that is being pursued to identify design changes that would mitigate the risk.

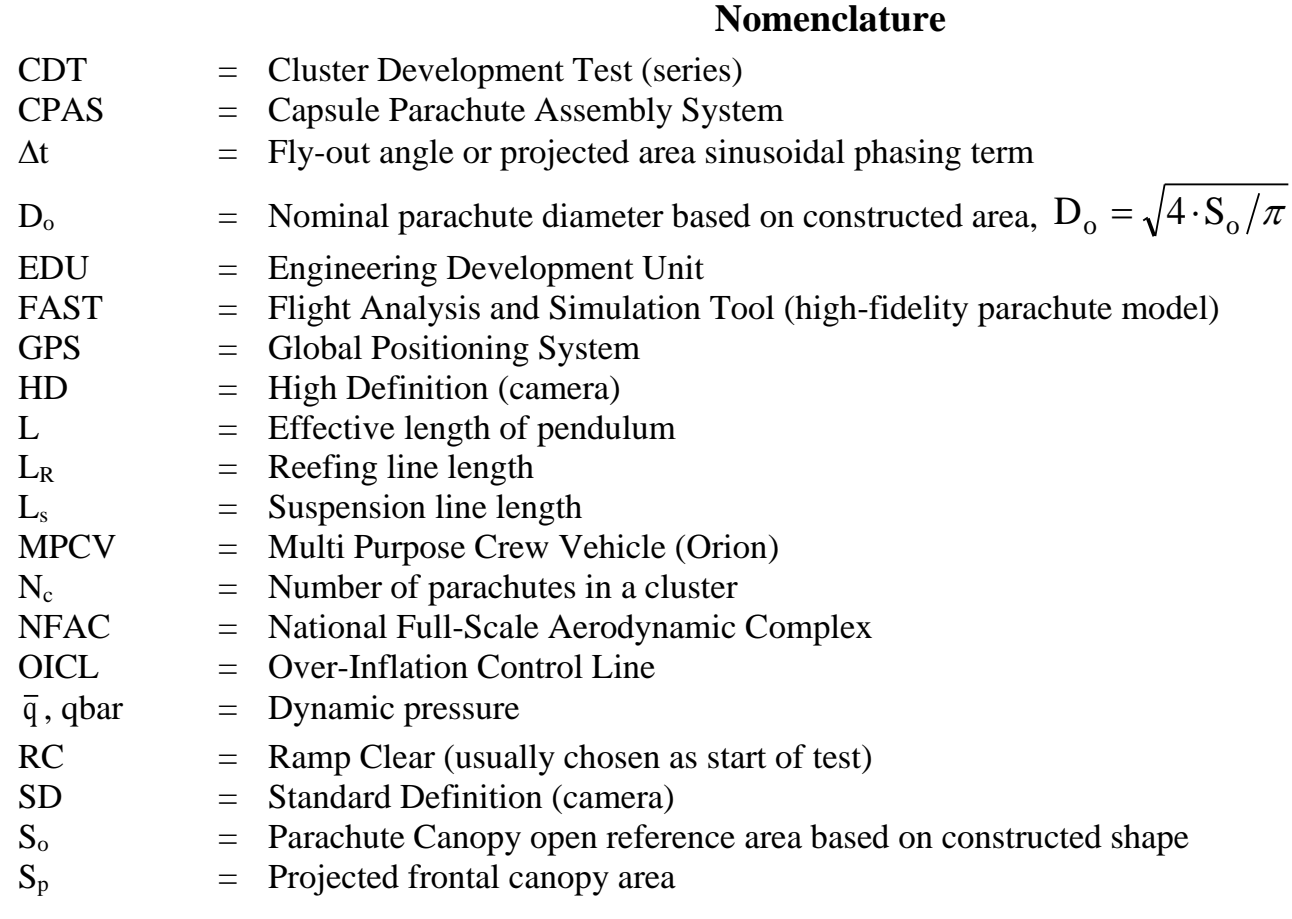

${ }^{1}$ CPAS Chief Engineer, Aeroscience Branch, NASA Johnson Space Center/EG3, AIAA Member.
${ }^{2}$ Analysis Engineer, Aeroscience and Flight Dynamics, 2224 Bay Area Blvd, Houston, TX, AIAA Member.

American Institute of Aeronautics and Astronautics 


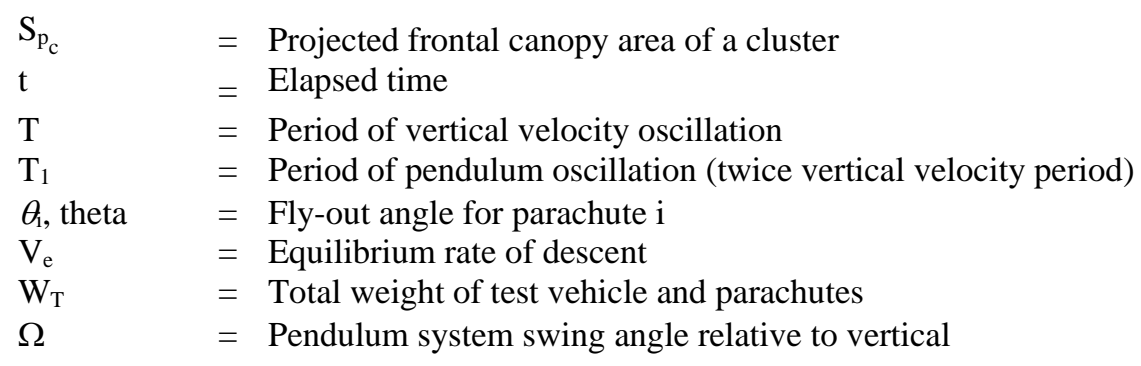

\title{
I. Introduction
}

\begin{abstract}
cluster of two or three $116 \mathrm{ft} \mathrm{D}_{\mathrm{o}}$ ringsail Main parachutes is intended to decelerate the Orion Multi Purpose Crew Vehicle (MPCV) to an ocean splashdown. The Capsule Parachute Assembly System (CPAS) is currently undergoing Engineering Development Unit (EDU) testing, where pendulum-like motion was encountered on multiple occasions. The pendulum oscillation was most prevalent for clusters of two canopies but may also occasionally occur when a cluster of three canopies align in a row.

Because this condition seems to grow in amplitude and maintain itself for a prolonged period, it is sometimes described as "swing amplification" to differentiate it from a simple gust response. This motion creates a large

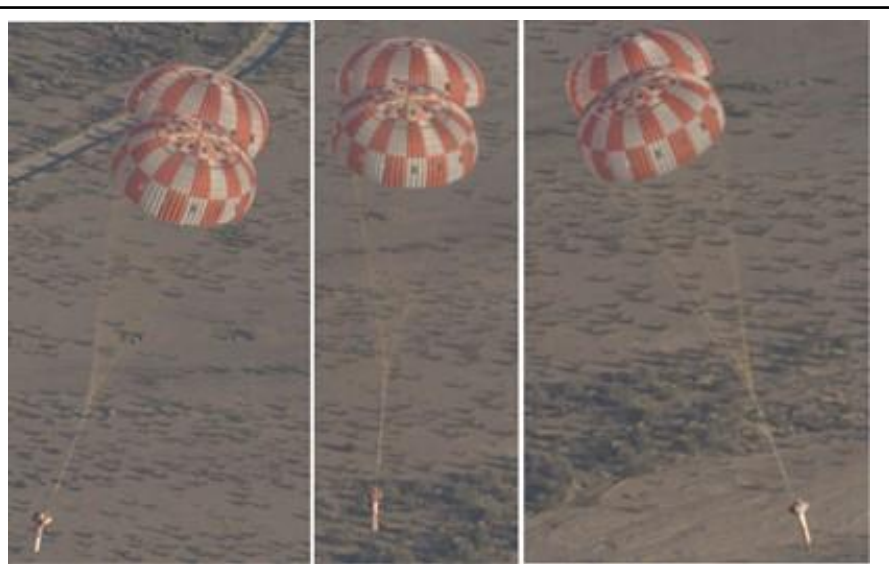

Figure 1. Pendulum motion under two Mains observed from chase helicopter during CDT-3-12.

horizontal velocity component and complicates roll attitude control, potentially leading to excessive landing conditions. An example of the pendulum swinging from Cluster Development Test (CDT)-3-12 is shown in Fig. 1.

The CPAS program developed a three phase plan to address the issue. Phase I of the plan consists of generating a first order empirical model to superposition the pendulum effect on the current terminal rate of descent performance model. ${ }^{1}$ Phase II is to update the parachute simulation capability to predict the likelihood and consequences of this phenomenon. The Pendulum Action Team (PAT) was assembled to perform this task. Phase III is to assess the cause of pendulum motion and determine a mitigation strategy including design changes. This task includes testing modified subscale canopies in both a wind tunnel and free flight. A previously scheduled full scale flight was conducted in parallel, where minor modifications to the Main canopies assessed.
\end{abstract}

\section{Phase I: Simple Pendulum Model}

A time-varying rate of descent model was previously developed by CPAS based on the observation that the instantaneous cluster projected area is directly proportional to the vertical velocity drag coefficient. ${ }^{2}$ However, this model assumes a symmetric cluster formation where each canopy creates an equal fly-out angle $(\theta)$ about a nearly vertical axis, as illustrated in Fig. 2. This model produces dispersed time histories of vertical rate of descent based on measured flight test data.

The simple model known as pendulum 1.0 was developed to estimate the additional velocity imparted on the vehicle based on the harmonic frequencies observed during flight tests. The pendulum swing angle $(\Omega)$ was defined the by angle made by the fly-out axis and the vertical, as shown in Fig. 3. 


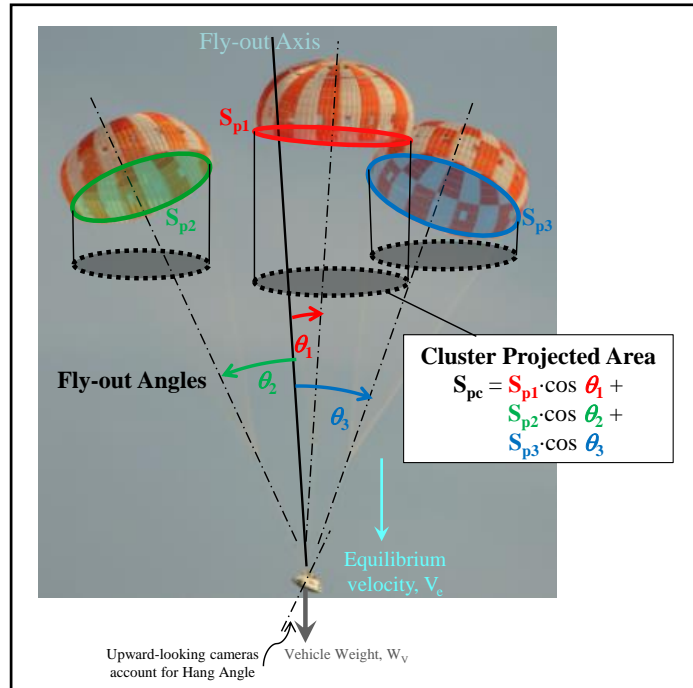

Figure 2. Symmetric formation assumed in rate of descent model.

The theoretical swing angle is a simple harmonic oscillator described by the pendulum frequency and the maximum amplitude $\left(\Omega_{\max }\right)$ as in Eq. 2 . The period of the swing angle is twice that of the vertical velocity component of the mass, as illustrated in Fig. 4. This relationship provides two redundant methods for determining the system frequency during flight; either indirectly through the swing angle, or from directly measured vertical velocity. Flight test data was also screened statistically for the characteristic "U-shaped" histograms seen in the simple pendulum data.
Time histories of the pendulum swing angle were made using photogrammetrics and vehicle attitude measurements. The vent locations of both canopies were tracked from onboard High Definition (HD) video and were calculated relative to the camera. The cameras had been installed with a known orientation relative to the test vehicle to account for the static hang angle under the Mains. The parachute locations were then transformed to the vehicle axis system. The vehicle attitude is measured by the NovAtel SPAN-SE (Synchronized Position Attitude \& Navigation) GPS/IMU. ${ }^{3}$ Once the parachute locations were determined in absolute (NED) coordinates, the swing angle could be computed.

The period of oscillation of a simple pendulum $\left(T_{1}\right)$ is based on the length of the pendulum $(L)$ and gravity $(g)$, as in Eq. 1.

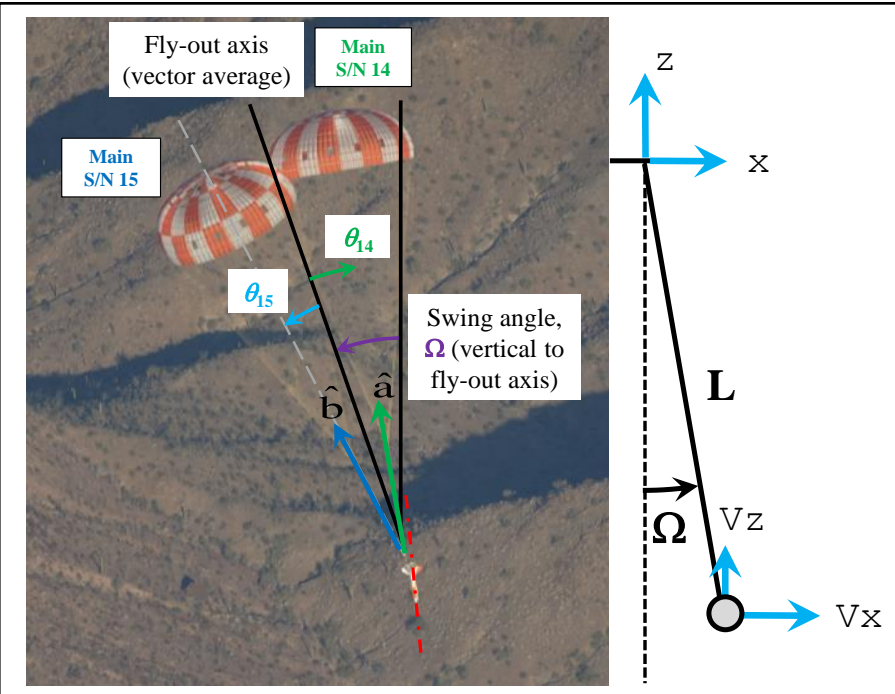

Figure 3. Definition of pendulum swing angle.

$$
\begin{gathered}
T_{1}=2 \pi \sqrt{\frac{L}{g}} \\
\Omega=\Omega_{\max } \sin \sqrt{\frac{g}{L} t}
\end{gathered}
$$




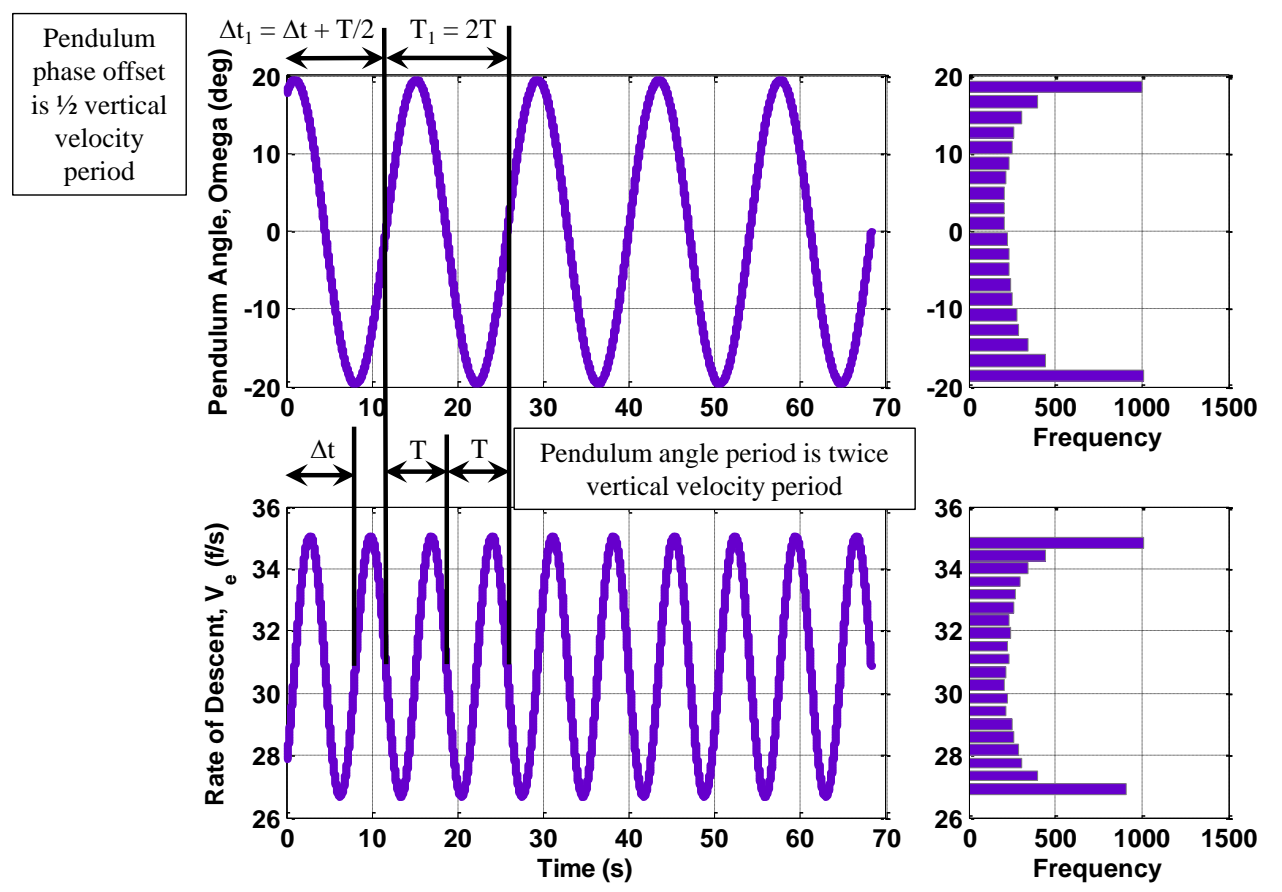

Figure 4. Relationship between pendulum swing angle and vertical velocity component.

\section{A. First Significant Pendulum: CDT-3-11}

CDT-3-11 involved the capsule shaped Parachute Test Vehicle (PTV). This system developed a significant pendulum oscillation about 230 seconds after ramp clear at an altitude of about 3,500 ft MSL. Ground camera footage and the rate of descent time history is shown in Fig. 5. An autocorrelation method determined the first order period of oscillation of the vertical velocity to be about 7 seconds.

The locations of the parachutes were transformed to an absolute frame as illustrated in Fig. 6. The vertical projection of the payload location relative to an axis between parachute skirts indicates motion mostly within a single plane perpendicular to the canopies. This geometry, in addition to the wind flow direction, is essential to determining the root cause of the swing amplification. The period of oscillation of the swing angle was
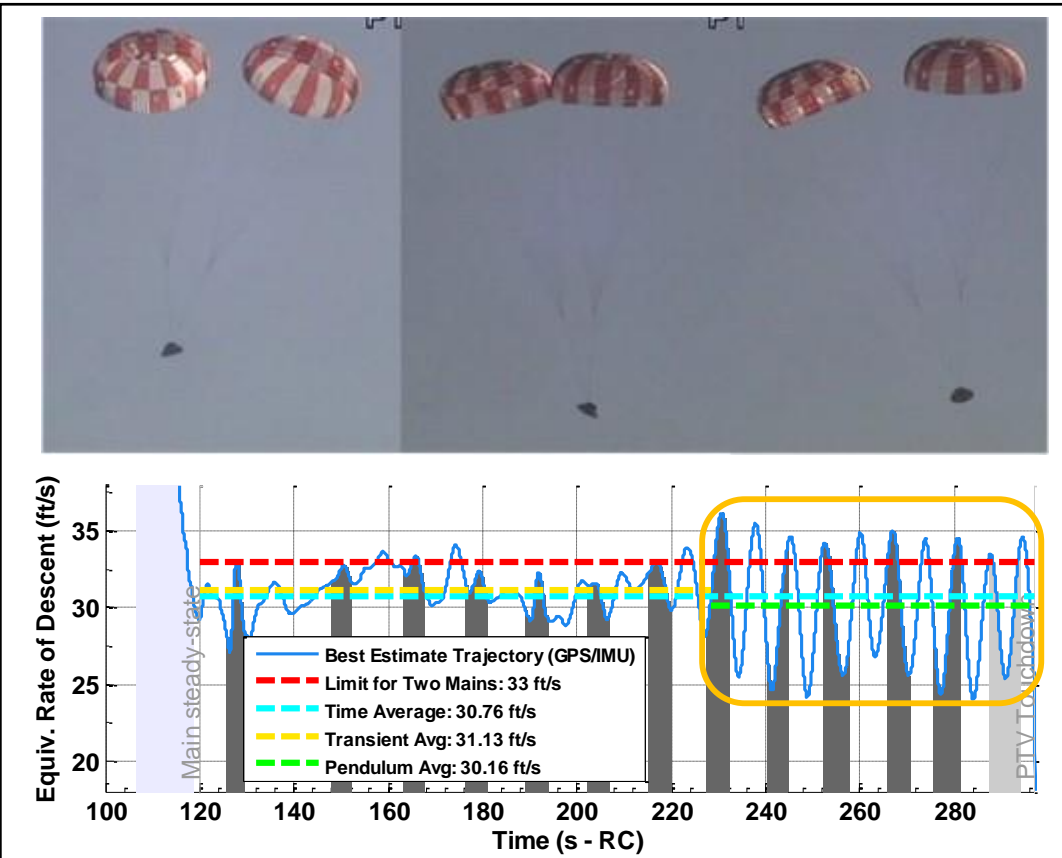

Figure 5. CDT-3-11 pendulum oscillation and rate of descent time history.

determined to be about 14 seconds from an autocorrelation function. As expected from the simple pendulum model, the period of the swing angle is almost exactly twice that of the vertical velocity. 

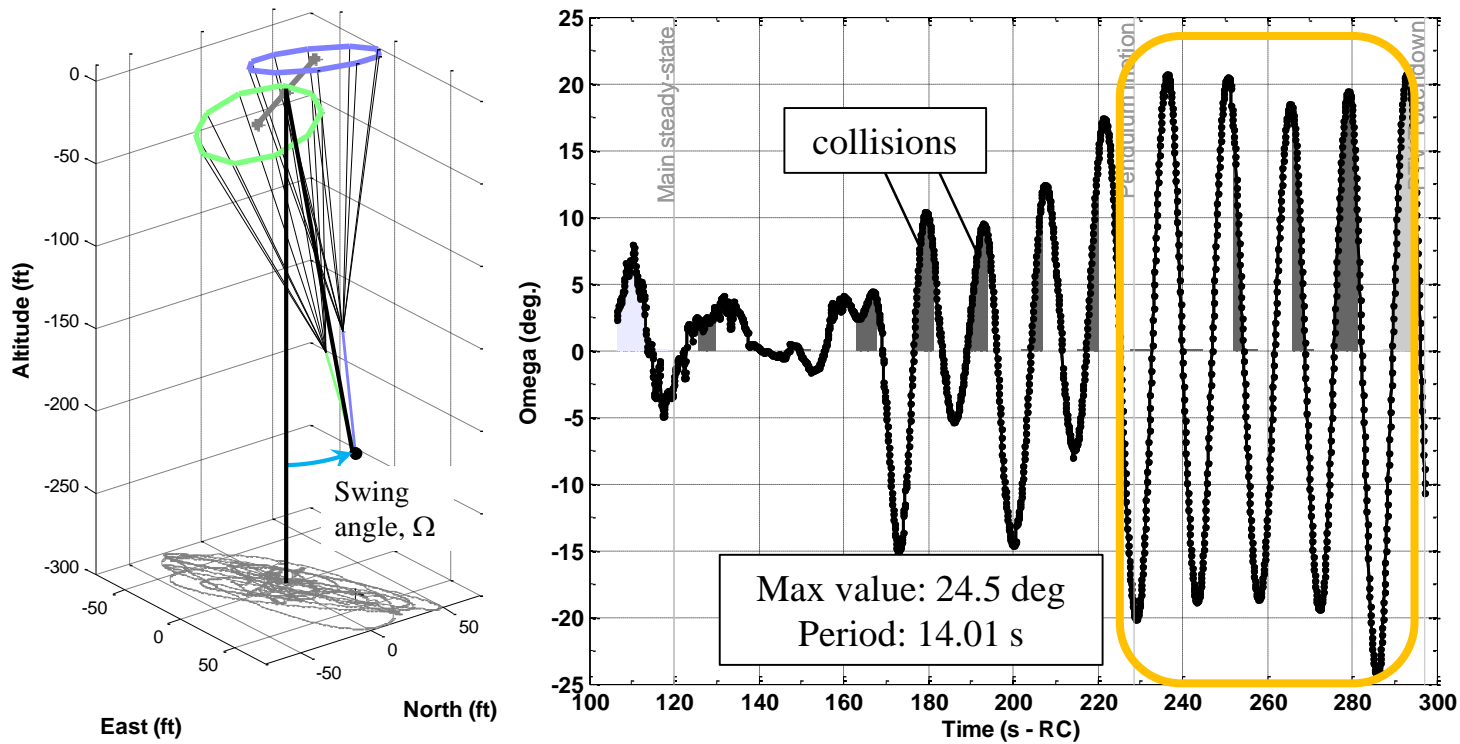

Figure 6. CDT-3-11 cluster geometry (left) and pendulum swing angle history (right).

This frequency corresponds to a pendulum length of about $163 \mathrm{ft}$, which is about three quarters the distance from the payload to the canopy skirts. This location was later confirmed to be the approximate barycenter of the system by filtering the motion of the payload and the canopies.

An examination of the plane of oscillation shows it to be in line with the primary wind direction during the altitude range under observation, as shown in Fig. 7. This seems to indicate that pendulum oscillation is correlated with winds along the "weak" cluster axis.
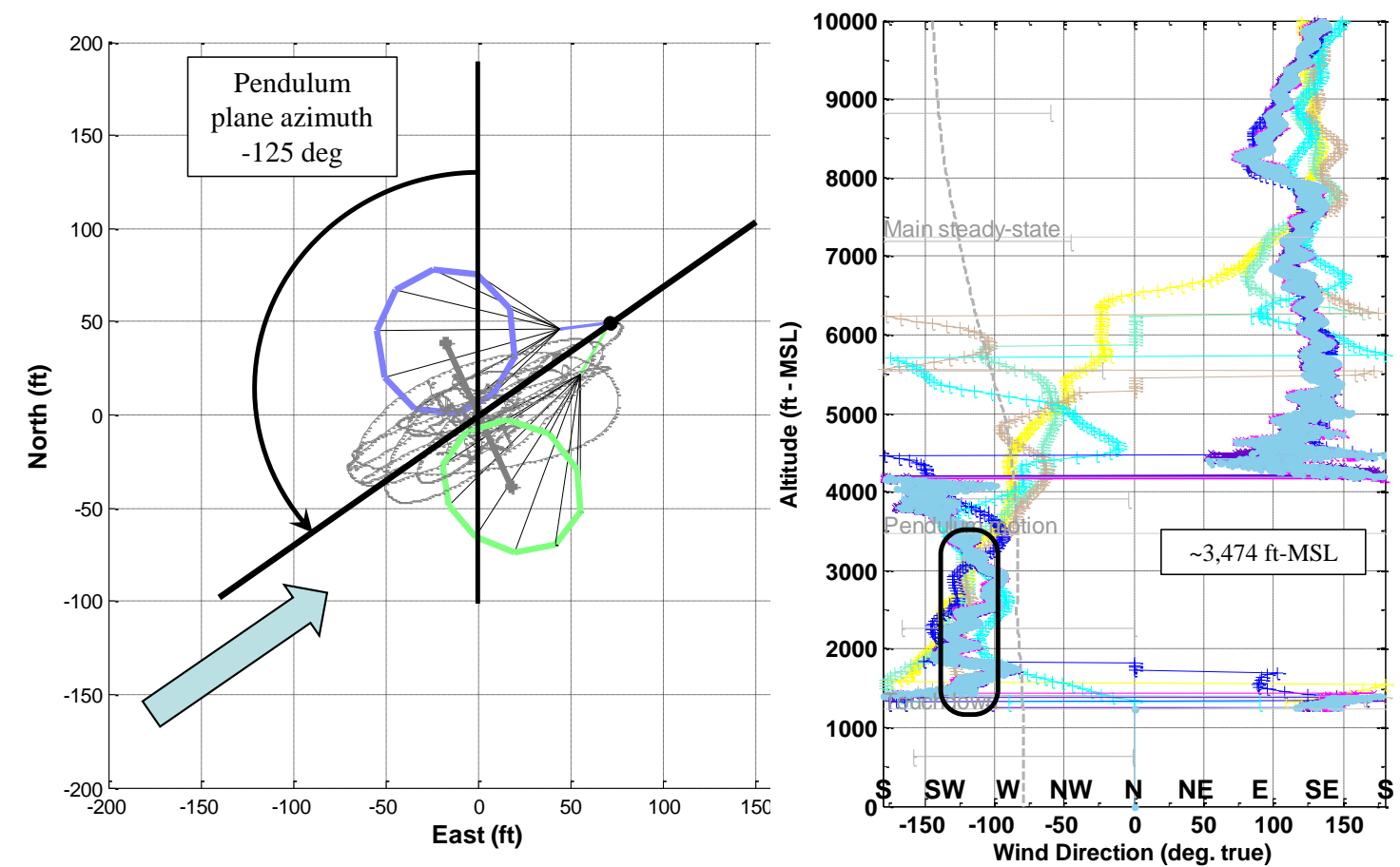

Figure 7. CDT-3-11 plane of oscillation and wind direction. 


\section{B. Second Significant Pendulum: CDT-3-12}

CDT-3-12 was a two-Main test using the dart-shaped Parachute Compartment Drop Test Vehicle (PCDTV). Experiencing swing amplification with this vehicle therefore seems to indicate it to be independent of forebody aerodynamics. Both test vehicles use a single-point attachment of the Main parachute risers, as will be used on the Orion vehicle. However, swing amplification was not observed on any of the five two-Main tests during Gen I or Gen II. Those earlier tests incorporated various differences in the Main canopy design but all used a flat platform suspended by harness legs. This may indicate a correlation between the stability of the payload attachment and the stability of the system, although later subscale tests were able to replicate pendulum motion with stable platforms.

The rate of descent is shown in Fig. 8, with the pendulum range circled. Statistics on the swing amplification for this test began about 206 seconds after ramp clear.

In order to speed up delivery of the Pendulum 1.0 model, the pendulum frequency and amplitude was determined exclusively from velocity information. The primary period of oscillation for the rate of descent was calculated as 7.3 seconds. System oscillation was later fully characterized when photogrammetric analysis was completed.

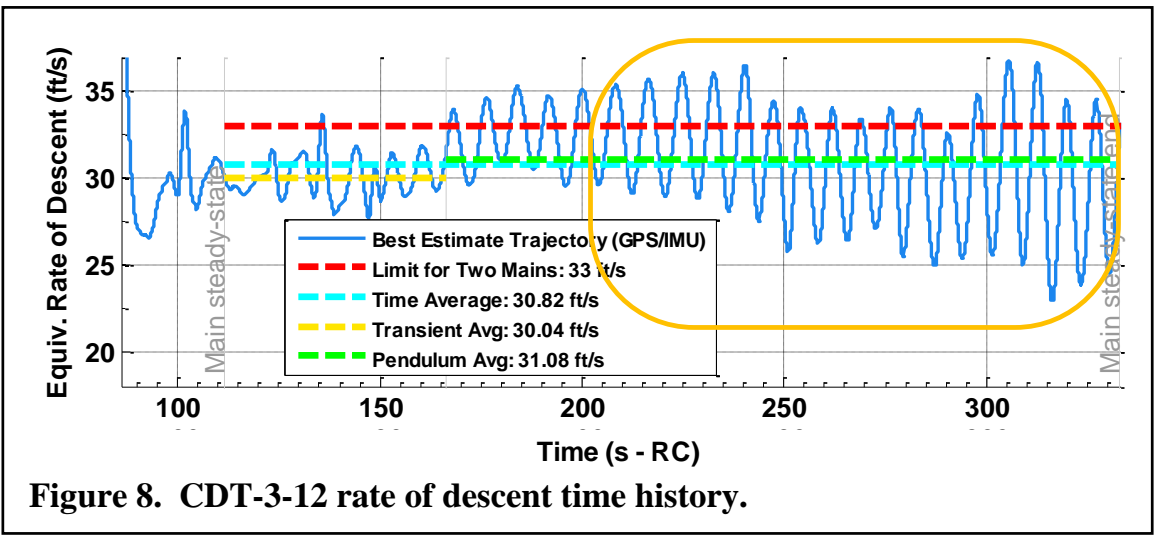

\section{Incomplete Pendulum Motion: CDT-3-8}

The significant swing amplification encountered during CDT-3-11 and CDT-312 prompted a close reexamination of previous tests for any evidence of pendulum motion. CDT-3-8 was a threeMain test where one of the canopies was modified for "flagging." That canopy could not sustain its weight and eventually fell below the vehicle, making this essentially a two-Main test.

A brief disturbance just before touchdown was originally attributed to surface effects and/or the riser of the flagging Main wrapping around the test vehicle. Upon closer inspection, this appears to be a case of pendulum motion which never had time
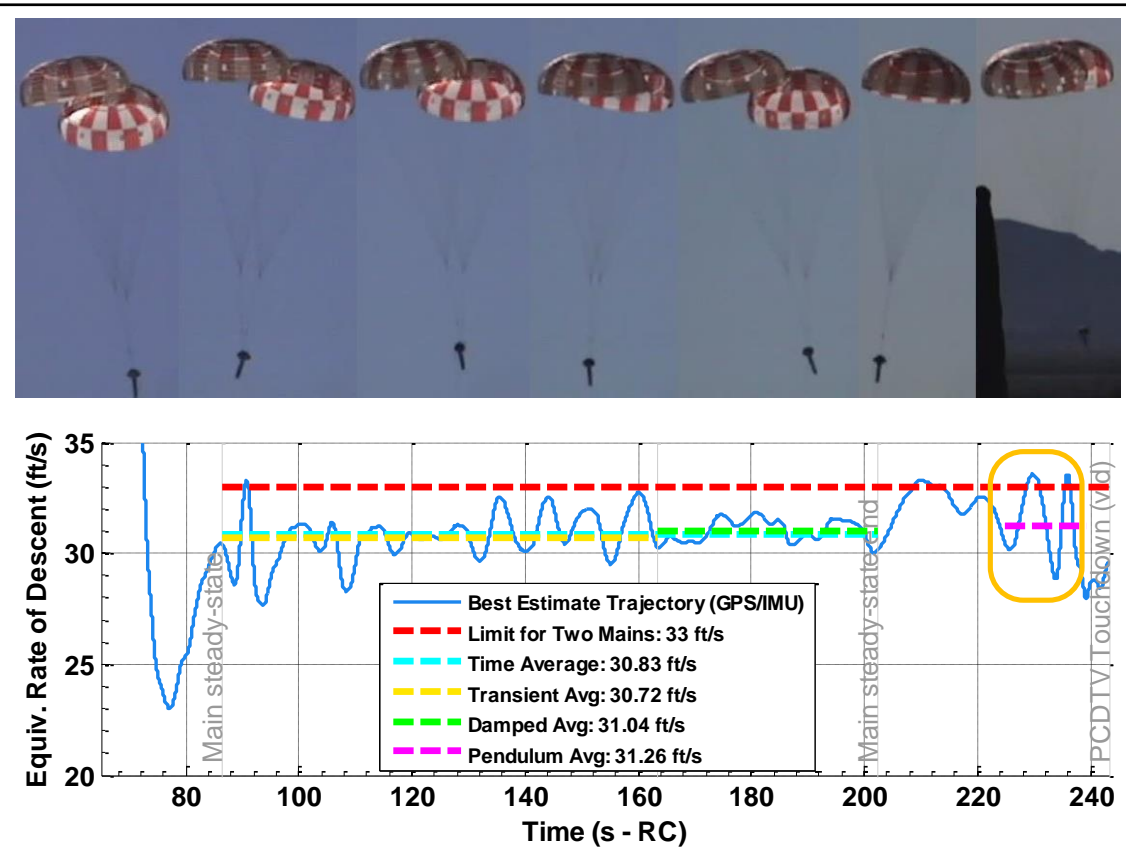

Figure 9. CDT-3-8 pendulum motion just before touchdown. to fully develop. This region is circled in Fig. 9.

The period of oscillation based on vertical velocity was computed as 15.8 seconds. However, these results were not included in the empirical model.

\section{Brief Three Main Pendulum Motion: CDT-3-7}




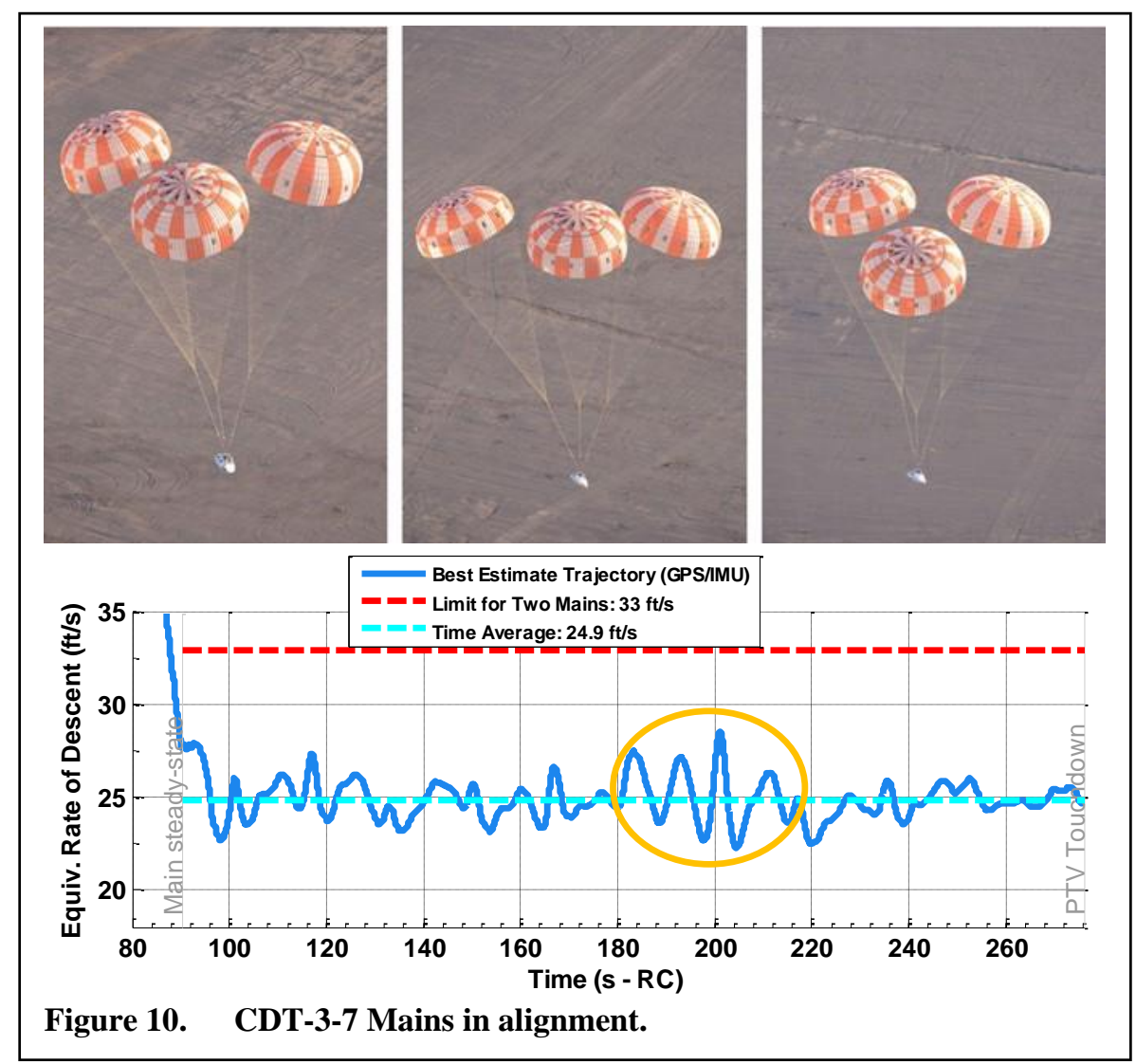

Three-Main flight tests were also assessed for pendulum motion. If pendulum motion were to start among two canopies, the third canopy would tend to damp that motion. However, it was determined from analysis of inertial velocities that CDT-3-7 briefly had a pendulum motion while all three canopies were forming a straight line, as shown in Fig. 10. Two complete pendulous cycles with a period of about 15 seconds were observed. Once the canopies re-aligned to a more triangular shape, the oscillations damped out.

Although these data could not be incorporated into the simple pendulum model, it does indicate that there is a potential for adverse cluster dynamics during splashdown. The model developed in Phase II is intended to simulate the potential for this behavior.

\section{E. Two-Main Modified Risers and OICL: CDT-3-15}

CDT-3-15 was a test previously scheduled to assess the EDU design. It was confirmed as a twoMain test once the pendulum issue became apparent. In order to maintain its schedule, only relatively simple modifications to mitigate pendulum effects were entertained.

The first modification was the reduction in riser length to more closely approximate the Apollo geometry. It was expected that this would better control the cluster formation.

The geometry is shown in Fig. 11. The relative distance from the payload to the barycenter is expected to remain approximately three-quarters of the distance to the skirt. Since the distance from the barycenter to the skirt is reduced, the amount of horizontal travel experienced by the canopies should be reduced, reducing the amount of enclosed air mass gained and lost through every oscillation cycle.

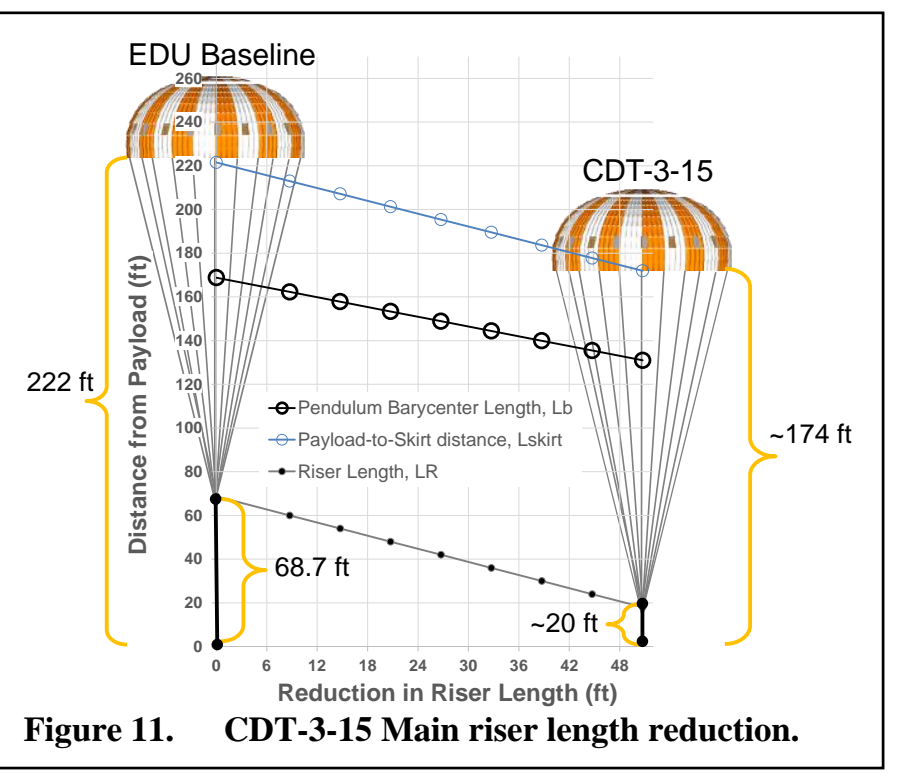


A relationship between canopy breathing and swing amplification is suspected. An Over-Inflation Control Line (OICL) is a simple method to restrict the canopy diameter. CPAS has previously used an OICL on two occasions. ${ }^{4}$ The first use (on test TSE-1A) used a length which was too long and did restrict the canopy dimensions. A better assessment of the canopy diameters using photogrammetrics allowed for an excellent OICL sizing on MDT-2-1. Because the EDU design has changed in both suspension line length and canopy porosity, a slightly different OICL length was determined by photogrammetric analysis of all available EDU tests.

Images from CDT-3-15 are shown in Fig. 12. The canopies slowly rotated around a central axis for majority of Main steady-state descent. Eventually, the system stopped rotating and transitioned to pendulum oscillations for the duration of the flight. Flight data are being evaluated to determine whether the cluster characteristics are more favorable than the baseline EDU design.

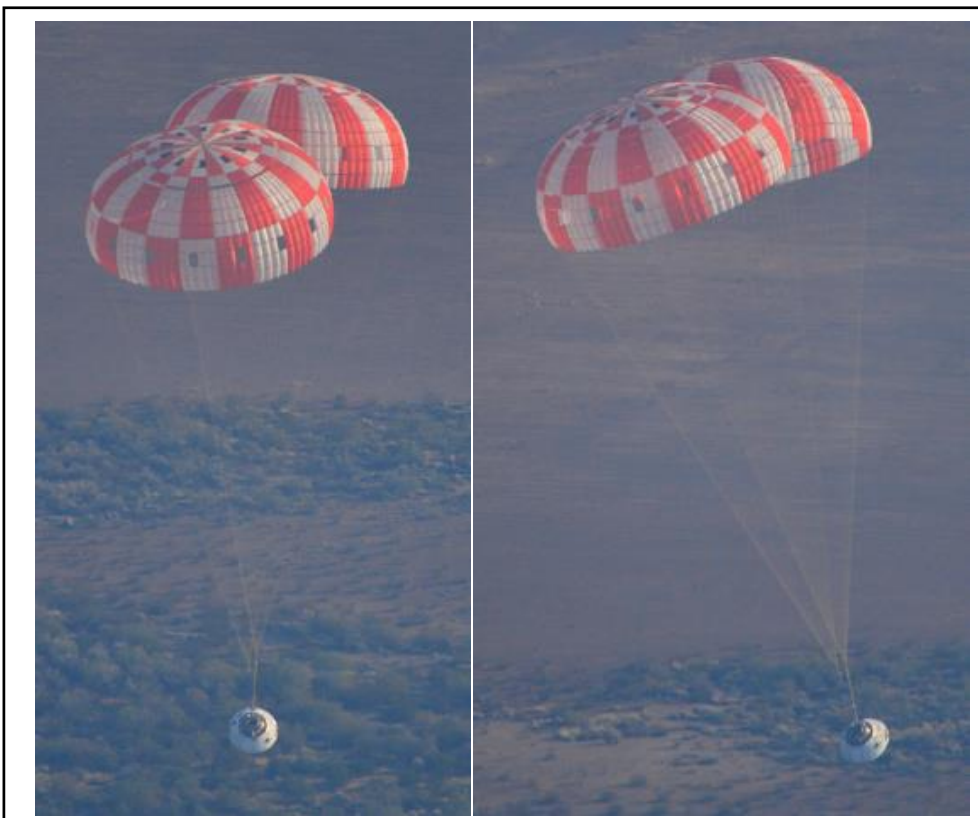

Figure 12. Despite the reduced riser length and OICL installation, CDT-3-15 exhibited signs of pendulum oscillation.

\section{Phase II: Improved Simulation}

The Pendulum Action Team is conducting several projects with the ultimate goal of fully simulating the complicated cluster dynamics which may result in swing amplification. A multi-disciplinary approach was taken to characterize the causes and potential mitigations of pendulum motion.

\section{A. Canopy Aerodynamic Models from Flight Test}

Knowledge of the parachute movement in flight and the measured riser loads allows for determining the aerodynamic coefficients of individual canopies. Each CPAS flight test records "best estimate" data for the ambient atmosphere, wind vectors, and payload trajectory. The positions of each canopy are measured relative to the payload using photogrammetry cameras fixed to the payload, as discussed in Ref. 2. It was therefore possible to determine the aerodynamic incidence angles on each canopy using a series of coordinate transformations. Recorded riser loads provided the axial force response history. These data were combined by the PAT into a database of aerodynamic coefficients for the given design flown.

\section{B. Modifications to FAST}

CPAS has transitioned to the Flight Analysis and Simulation Tool (FAST) for both preflight predictions and posttest reconstructions. The high-fidelity parachute model in FAST improves upon legacy tools in modeling individual canopies, rather than as a composite canopy. This architecture allows for the independent movement of canopies, similar to actual flight.

Several two-Main flight tests were reconstructed by the PAT using FAST. Each test was initiated at as the Mains inflated to full open. Atmospheric and wind data were read in for each test as a function of altitude. Aerodynamic forces and moments on each canopy were simulated based on the aerodynamic database. Characteristic pendulum motion was replicated, including maximum swing angle, period, and fly-out angles. A test where pendulum motion did not occur (CDT-3-2) was also reconstructed as a control. 


\section{Rigid-Body CFD}

The stability of various full open canopies was predicted by rigid body, static Computational Fluid Dynamics (CFD) analysis. Inflated grids were generated based on shapes determined from the CAnopy Loads Analysis (CALA) code for the given gore layout. The OVERFLOW code was employed to determine aerodynamic coefficients of each rigid canopy shape. Material permeability was not modeled. A series of legacy Apollo ringsail parachute designs were first simulated to validate the approach. CFD results were compared with observed Apollo system oscillation angles recorded in NVR-3722.

Next, a series of cases were run to evaluate the static stability of the Gen I CPAS Main canopy as well as the design changes incorporated into the current EDU canopy. Angle of attack sweeps were run on potential design modifications, based on expert recommendations and incremental results. An examination of the flow-fields shows a dependence on the size and position of geometric porosity. An example comparison is shown in Fig. 13.

A limited subset of cases were run with two canopies in close proximity to determine the incremental effect on static aerodynamics of mutual interference. An evaluation of aerodynamic coefficients from design changes was used to plan

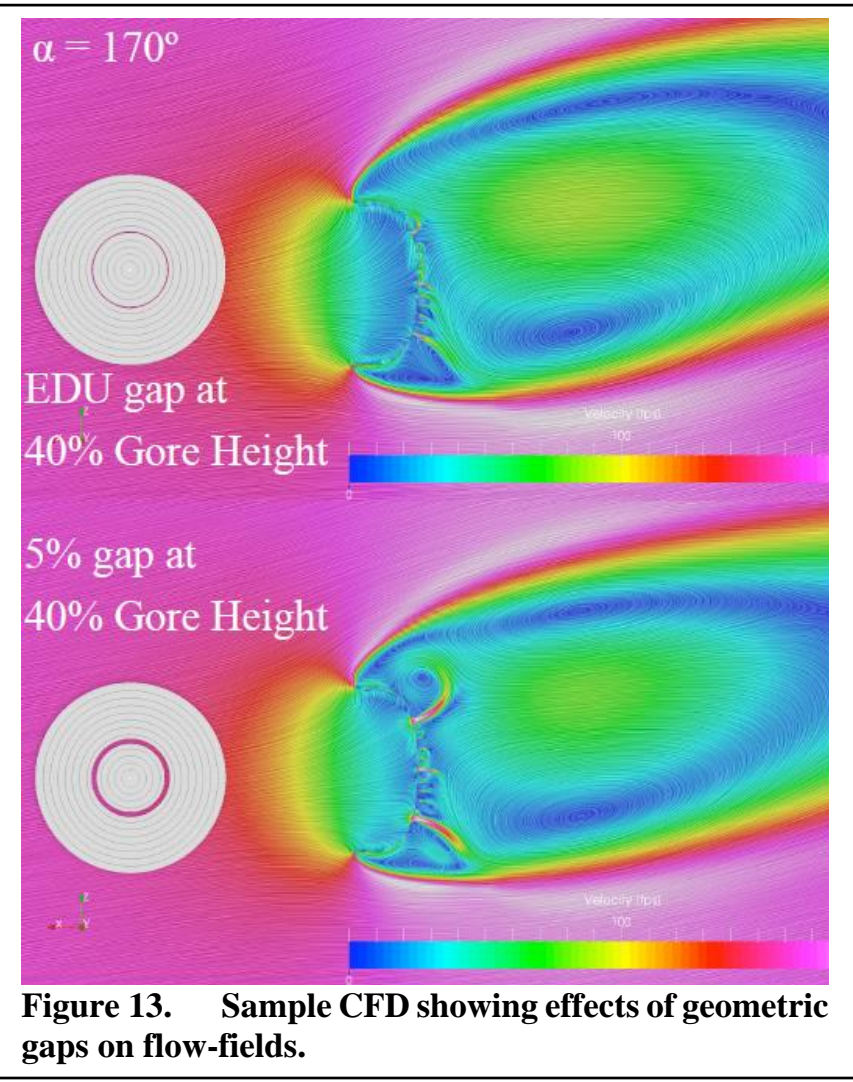
the wind tunnel test matrix.

\section{Phase III: Root Cause and Mitigation}

In order to evaluate potential design changes to the CPAS Main parachutes, the program decided to examine subscale canopies, which could be constructed and tested more rapidly than full size canopies. Airborne Systems constructed a series of canopies scaled to $35 \%$ with the baseline design and various potential changes. This size allowed for use in the $80 \times 120 \mathrm{ft}$ wind tunnel at the NASA Ames Research Center. It also allowed for free flight tests from small aircraft.

\section{A. Ames $80 \times 120 \mathrm{ft}$ Wind Tunnel Test}

Approximately two weeks of testing were conducted at the National Full-Scale Aerodynamic Complex (NFAC). A primary objective of the subscale wind tunnel test was to down-select to two canopy configurations for follow-on air drop testing. Another objective was to gather single-canopy static and dynamic aerodynamic data for each configuration. In general, it was expected that canopy stability could be achieved at the expense of drag performance. Single canopy aerodynamic coefficients determined using photogrammetric and loads data using methods based on NFAC experience.$^{5,6,7}$ Static aerodynamic data was gathered using a three-tether and load cell system attached to the parachute vent, coupled with axial measurements, as shown in Fig. 14. Dynamic aerodynamic data was gathered using photogrammetry of the free-flying parachute following aerodynamic data extraction. 
Thirteen configurations were tested. Aside from the baseline EDU design, potential modifications included an OICL and various geometric porosity adjustments. A total of 391 test data points were gathered across all configurations at various angles of attack or free-flight conditions. Although it was not possible to evaluate all of the test data in time for the subscale flight tests, qualitative assessments of free-flight dynamics allowed for flight test hardware decisions.

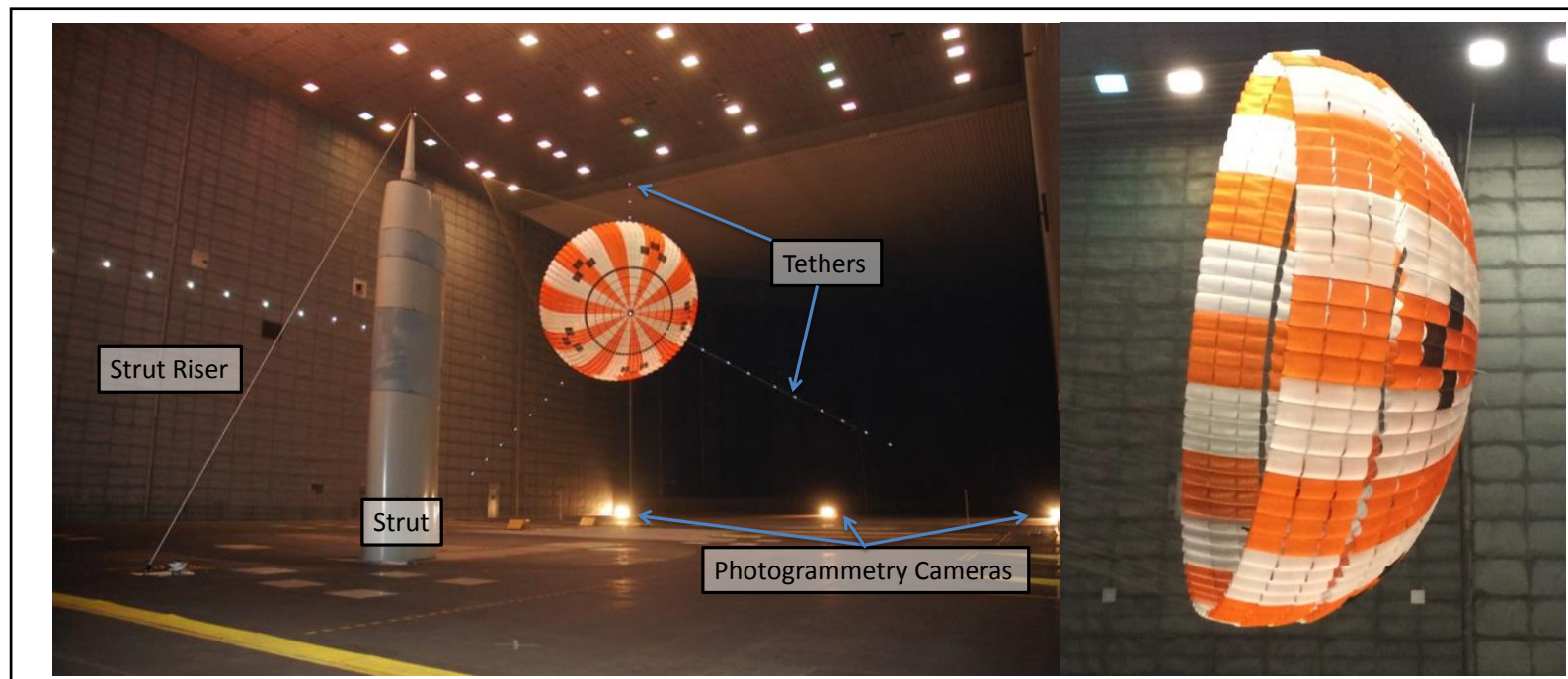

Figure 14. Candidate Main design being evaluated at Ames 80×120 ft wind tunnel.

\section{B. Froude Number Scaled Flight Test}

Subscale flight tests were conducted over two separate weeks at a skydiving range in Eloy, Arizona. The concept of operations was to extract one or two payloads from the Short SC.7 Skyvan twin-turboprop on every flight. The appropriate payload weight was determined based on matching the Froude number of the full open Main. Test vehicles were developed for both single and cluster tests, each with an avionics and camera suite to allow complete system reconstructions. Aside from the candidate geometric porosity, configurations were also modified with short or long riser lengths and the presence or absence of an OICL. Initial single-canopy tests were run to determine OICL length for each design based on photogrammetric analysis.

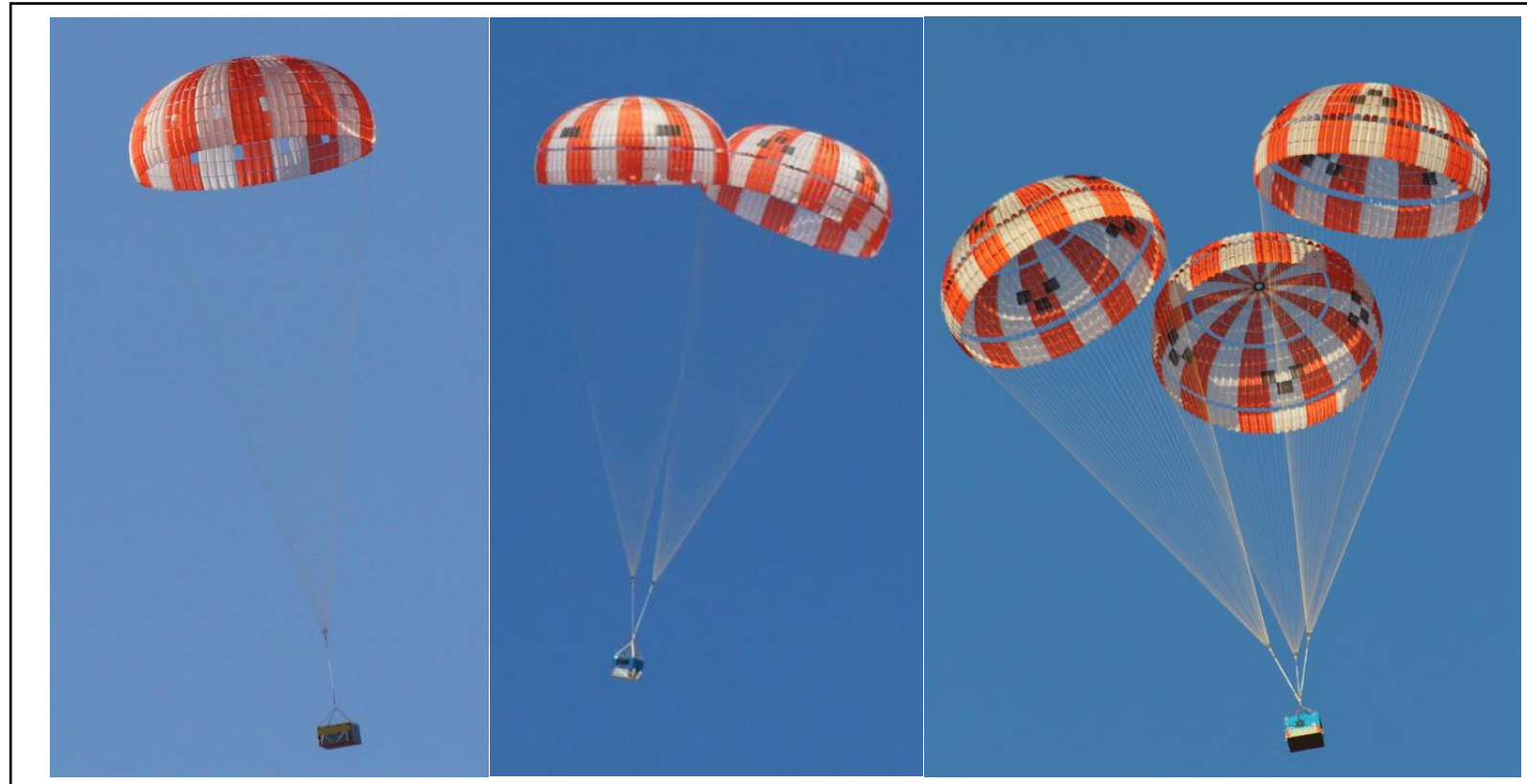

Figure 15. Sample Froude number scaled flight tests with candidate configurations.

American Institute of Aeronautics and Astronautics 
The bulk of flights were in clusters of two Mains in order to evaluate pendulum motion. A small number of tests were run with a cluster of three Mains to evaluate nominal system performance. Some tests incorporated first or second stage reefing to ensure the new configurations did not have adverse effects on reefed canopies. Typical tests are shown in Fig. 15.

Many two-Main flights exhibited pendulum motion, while others did not. A close evaluation of the relative wind conditions for each flight should help ascertain the probability of pendulum dynamics. Flight data are still being evaluated and will eventually be used to recommend a final full scale configuration.

\section{Conclusion}

A significant pendulum motion characterized as swing amplification has been observed on CPAS two-Main flight tests. Close inspection of previous tests shows this phenomenon indicates possible pendulum motion even during three-Main tests, if the canopies are in a straight line. The consequences of pendulum motion at landing can be severe. Therefore, a three phase plan was created to study this problem and suggest mitigations.

\section{Acknowledgments}

[The contributions of each PAT member will be listed, and/or they can be added as co-authors.] Photogrammetric data was reduced by David Bretz and others with the JSC-KX Image Science \& Analysis Group.

\section{References}

\footnotetext{
${ }^{1}$ Ray, E., “A Symmetric Time-Varying Cluster Rate of Descent Model,” 23rd AIAA Aerodynamic Decelerator Systems Technology Conference, Daytona Beach, Florida, March 2015, submitted for publication.

${ }^{2}$ Ray, E. and Bretz, D. R., "Improved CPAS Photogrammetric Capabilities for Engineering Development Unit (EDU) Testing," $22^{\text {nd }}$ AIAA Aerodynamic Decelerator Systems Technology Conference, Daytona Beach, Florida, March 2013, AIAA paper 20131258.

${ }^{3}$ NovAtel, Inc., "SPAN-SE," NovAtel, Inc. web site [online], February 2010, URL: http://novatel.com/Documents/Papers/SPANSE.pdf [cited 23 March 2010].

${ }^{4}$ Morris, A. L., Bledsoe, K. J., Fraire, U., et al., "Summary of CPAS Gen II Testing Analysis Results," $21^{\text {st }}$ AIAA Aerodynamic Decelerator Systems Technology Conference and Seminar, Dublin, Ireland, May 2011, AIAA paper 2011-2585.

5 Schoenenberger, M., Queen, E. M., and Cruz, J. R., "Parachute Aerodynamics from Video Data," $18^{\text {th }}$ AIAA Aerodynamics Decelerator Systems Technology Conference, Munich, Germany, AIAA paper 2005-1633.

6 Tanner, C. L., Clark, I. G., Gallon, J. C., and Rivellini, T. P., "Aerodynamic Characterization of New Parachute Configurations for Low-Density Deceleration," 22 nd AIAA Aerodynamic Decelerator Systems Technology Conference, Daytona Beach, Florida, March 2013, AIAA paper 2013-1315.

${ }^{7}$ Gonyea, K. C., Tanner, C. L., Clark. I. G., Kushner, L. K., Schairer, E. T., and Braum, R. D., “Aerodynamic Stability and Performance of Next-Generation Parachutes for Mars Descent," 22 $2^{\text {nd }}$ AIAA Aerodynamic Decelerator Systems Technology Conference, Daytona Beach, Florida, March 2013, AIAA paper 2013-1356.
}

American Institute of Aeronautics and Astronautics 